\title{
Interactive comment on "Importance of the advection scheme for the simulation of water isotopes over Antarctica by general circulation models: a case study with LMDZ-iso (LMDZ5a revision 1750)" by Alexandre Cauquoin and Camille Risi
}

\section{Anonymous Referee \#2}

Received and published: 27 October 2017

Cauquoin and Risi have investigated the effect of the advection scheme on the simulation of the water isotopes and temperatures over Antarctica. By conducting sensitivity tests with the LMDZ-iso model, they have concluded that the diffusivity of the advection scheme on the horizontal plane is crucial to simulate the water isotopes and temperatures over Antarctica. The paper is well written and structured. However, the scientific finding is very thin. The unacceptable level of the diffusivity property of the upstream scheme is a well-known fact and the sensitivity results are not surprising.

Printer-friendly version

Discussion paper 
Therefore, I cannot recommend this manuscript for publication at this stage. As the authors argued, the diffusive property of AGCMs is attributable to the performance of the advection scheme, but it is a much higher level than that of the upstream scheme. I believe that the upstream scheme is no longer used in current state-of-the-art AGCMs. Even the van Leer scheme is known as a diffusive scheme and using a less diffusive scheme is a challenging task. Actually, there are a number of more sophisticated advection schemes, but most of them are computationally expensive. Therefore, one may consider that the van Leer scheme is a reasonable choice in terms of accuracy and computational cost. I suggest that the authors should try more sophisticated schemes than the van Leer one and compare results in terms of accuracy and computational cost. Presumably, the computational cost is an important issue for paleoclimate studies, because they require very long-term simulations. Hourdin and Armengaud (1999) might be a good reference paper, in which not only the upstream scheme and van Leer schemes, but also other sophisticated schemes were tested based on the LMDZ model. If such advection scheme tests are done for the water isotopes and discussion is made from the paleoclimate view, that would be an attractive paper.

\section{Minor comments:}

\section{P1. L13: "good" is ambiguous.}

P3, L1-L3: I cannot understand what this sentence means.

P5, L7-9: "The fact that the advection. . . is not through temperature" is not fully convincing.

P6, L2-3: "The excessive diffusion ... but is not the main cause" should be removed from Conclusion, because "its main cause is beyond of the scope of the study" (P4, L28).

Printer-friendly version

Discussion paper

Reference:

Hourdin, F., and A. Armengaud, 1999: The use of finite-volume methods for atmo- 
spheric advection of trace species. Part I: Test of various formulations in a general circulation model. Mon. Wea. Rev., 127, 822-837.

Interactive comment on Geosci. Model Dev. Discuss., https://doi.org/10.5194/gmd-2017-178, 2017. 